\title{
The Relationship between Implant Size and Success Rate in Computer Guided Implant Surgery
}

\author{
Mohammed Abu Younis, Raéd O. Abu Hantash
}

Al-Quds-University, Jerusalem, Palestine

Correspondence to: Mohammad Abu Yunis, mabuyunis@staff.alquds.edu

Keywords: Guided Surgery, Surgical Template, Accuracy, Implants Dimension

Received: October 18, $2020 \quad$ Accepted: December 27, $2020 \quad$ Published: December 30, 2020

Copyright $\odot 2020$ by author(s) and Scientific Research Publishing Inc.

This work is licensed under the Creative Commons Attribution International License (CC BY 4.0).

http://creativecommons.org/licenses/by/4.0/

\section{(c) (i) Open Access}

\section{ABSTRACT}

Guided implant surgery gained acceptance and credit in implant dentistry. The aim of this study is to find out and evaluate the relationship between implant size and success rate following computed tomography scan guided implant surgery. Sixty-four (36 females, 28 males) completely and partially edentulous patients aged between 42 and 65 years old were participated and fulfilled the inclusion criteria of the study. Two hundred and seventeen dental implants (length: 10,11.5, and $13 \mathrm{~mm}$; Diameter: $3.3,3.75$, and $4.2 \mathrm{~mm}$ ) were placed using computed tomography scan guided implant surgery, 117 implants were placed in the maxilla, while 100 implants were placed in the mandible. The implants were evaluated for clinical parameters at 2,4 , and 12 weeks intervals after installation. The overall success rate was $95.4 \%$. Clinical stability of the implant size (Diameter $3.3 \mathrm{~mm}$, Length $10 \mathrm{~mm}$ ) was the least as compared to other dimensions $(P \leq 0.05)$. Medium and large size implants (Diameter 3.75 and $4.2 \mathrm{~mm}$, Length 11.5 and $13 \mathrm{~mm}$ ) were the highest success rate in implant surgery. Implants width (diameter $3.3 \mathrm{~mm}$ ) and length $(10 \mathrm{~mm}$ ) are best avoided in guided computed tomography surgery as compared with other implant sizes especially in atrophic bony areas. Computer-guided implant surgery remains exciting. Watchful planning and precise stability of the surgical guide are mandatory for successful protocol. Further investigations should be performed to assess such technique among dental patients from satisfactory point of view. Intensive cooperation between dentists and software engineering discipline should be aimed to maximize the success of such technique.

\section{INTRODUCTION}

Guided surgery is a flapless procedure that offers a more precise, predictable and safe procedure with minimal postoperative discomfort and pain in implant treatment [1]. Such procedure using techniques 
derived from three-dimensional oral implant planning software, of both surgical templates for flapless surgery and dental prostheses for immediate loading, is a very preferable treatment choice [2]. Immediate loading can be defined as occlusal or nonocclusal restoration of implants on the same day the implants were inserted [3] or within the first two days following implant insertion, while conventional treatment returned to complete the restoration after 3 to six months [4]. The longer the implant, the better the primary stability. Implants shorter than $10 \mathrm{~mm}$ present a high risk of failure [5].

The mean survival rate of computer guided surgery is $97.2 \%$ with mean marginal bone loss of 1.45 $\mathrm{mm}$ in $1-4$ years follow up [6]. Surgical guidance helps the operator in installing the implant in the most preferable position and angulations to the final restorations [7]. Complete guided implant surgery can help operators in placing dental implants for rehabilitation of the edentulous maxilla without extraction of impacted teeth [8].

Patients exposed computer assisted implant surgery felt less pain and were more satisfied than conventional implant patients [9]. Computer guided implant surgery depends upon careful planning and perfect stability of the surgical templates [10]. Good bone quality, adequate thickness of keratinized gingiva and high primary stability are key factors for successful guided implant surgery [11]. Different computer guided implant surgery procedures are available with inaccuracy of $\pm 2.0 \mathrm{~mm}$ smaller than for nonguided surgery [12]. Existing dental implants in an overdenture can be used to fixate a surgical guide to place more implants to convert existing overdenture into fixed prosthesis [13]. Flapless implant surgery has a survival rate (96.5\% to $97.9 \%)$ comparable to conventional flap techniques [14]. Computed guided implant placement is useful and suitable approach for rehabilitation of edentulous maxilla without the extraction of impacted canines [15].

Computer-guided planning and placement of dental implants has become broadly used and has been considered to be a rather reliable tool for implant positioning without endangering the adjacent anatomical structures [16].

Computer-generated planning permits for improved imagining of bone morphology prior to the positioning of implants and improves the construction of implant supported restorations according to a predictable planning of implants [17].

In this context, the literature lacks valid studies of the relationship between implant size (length and width) and success rate in computer guided implant surgery. Therefore, this prompted the conduction of the current study in order to cast the light on this issue.

\section{MATERIALS AND METHODS}

The study population consisted of Sixty-four (36 females, 28 males) (Table 1) completely and partially edentulous patients aged between 42 and 65 years old were participated and fulfilled the inclusion criteria of the study (Table 2).

The patients were referred by general practitioners to a specialized Guided Implant Surgery Center in Jerusalem-Palestine in the period between 2012-2016. This center receives dental implant patients from the whole country.

Each patient was a given a brief explanation of the investigation and the procedures to be undertaken. An informed consent was obtained from each patient before implant surgery. To be included in the study, the recruited patients must be 18 years old or above in order to receive dental implant treatment and must be indicated to receive dental implant therapy.

Table 1. Distribution of study participants according to gender.

\begin{tabular}{cc}
\hline Gender & Number and percentage \\
\hline Females & $36(56 \%)$ \\
Males & $28(44 \%)$ \\
Total & 64 \\
\hline
\end{tabular}


Table 2. Distribution of participants according to their age.

\begin{tabular}{cc}
\hline Age (Years) & Number and percentage \\
\hline Below 45 & $20(31 \%)$ \\
Between $45-50$ & $33(51 \%)$ \\
Above 51 & $24(38 \%)$ \\
Total & 64 \\
\hline
\end{tabular}

The prosthetically guided maxillofacial surgery computer-aided design/computer-aided manufacturing surgical protocol was applied to sixty-four (partially and completely edentulous) patients between September of 2012 and March of 2016.

Prosthetically guided surgery computer-aided procedure involved four steps: 1 ) the virtual planning of the surgical treatment, 2) planning of edentulous site in the patients' casts (area where dental implants are going to be installed, 3) computer-aided design/computer-aided manufacturing and rapid prototyping procedures for the design and manufacture of the customized surgical devices, and 4) the surgical procedure itself. Virtual Planning began with the acquisition of a high-resolution tomographic scan of the patient's mouth. Computed tomographic imaging was performed using a multidetector computed tomography scanner (Carestream Dental Cone Beam Computed Tomography 3-D CBCT with CS 9300 and CS 8100-Kodack, New York, USA) for both the patients' mouth and the dental casts. All patients were asked to do CT Scan for their jaws, after making impressions in the dental clinics. Impressions were scanned to create digital models. Surgical templates were fabricated to suit every case in the study population regarding, number, diameter and length of dental implants used.

Surgical phase implants success was assessed according to the first three points of Albrektsson et al. (1986) who suggested the following criteria to judge the success of dental implants as:

1) A stable dental implant that lacks mobility.

2) Radiographic examination reveals no radiolucency around the installed dental implant.

3) The absence of pain, paresthesia, infection or any signs and symptoms complained by the patient.

4) The radiographic vertical bone loss is less than $0.2 \mathrm{~mm}$ per year, after one year of function.

5) The implant should accomplish the above mentioned guidelines with success rate of $85 \%$ at a 5 -year follow up and $80 \%$ at the end of a 10 -year period [18].

The null hypothesis was that in patients treated with guided implant surgery, no relationship could be established between implants' dimensions (length and diameter), and patients' parameters (age and gender) and the success of implant therapy.

This study was reviewed and ethically approved by the deanship of dental school at Al-Quds University.

\section{RESULTS}

Sixty four patients ( 36 were females and 28 were males) participated in the current study and received dental implants using guided implant technique.

A total of 217 implants were installed using computer guided surgical technique and three diameter dimensions namely: $(3.3,3.75$ and $4.2 \mathrm{~mm})$ were used and three implants length $(10,11.5 \mathrm{and} 13 \mathrm{~mm})$ were also used, and their distribution according to site and size is clarified in (Table 3 ).

According to the site of implant insertion; 117 implants were placed in the maxilla, while $100 \mathrm{im}$ plants were placed in the mandible. The implants were evaluated for clinical parameters at 2, 4, and 12 weeks intervals after installation, 6 maxillary implants and 4 mandibular implants failed to osseointegrate (Table 4).

The overall success rate was $95.4 \%$ (10 implants failed out of 217). According to the diameter dimension 8 implants ( $3.3 \mathrm{~mm}$ in diameter) were failed to osseointegrate during the study. Meanwhile, $9 \mathrm{im}$ - 
plants (10 $\mathrm{mm}$ in length) were also, failed to ossentegrate as noticed in (Table 5).

Clinical stability of implants dimensions (Diameter $3.3 \mathrm{~mm}$, Length $10 \mathrm{~mm}$ ) was the least as compared to other dimensions. Oneway ANOVA in SPSS (Statistical Package for the Social Sciences, version 20.0, Inc., Chicago, IL, USA) was used to compare the differences in implant failure according to implant parameters (length, diameter, and position). The results revealed that there is a significant effect between implant diameter as well as length and implant failure $(P<0.05)$, while anatomic position has no significant difference (Table 6).

In this context, patients' parameters (age and gender) were also studied using Oneway ANOVA, and the results showed that there are no significant differences between patients age and gender and their effect on implants failure $(P$ value $>0.05)$ (Table 7$)$.

\section{DISCUSSION}

Patients and dentists seek new effective treatment options to replace conventional removable prosthodontic treatment. Dental implants provided the answer and the alternative in many cases, and have emerged as a viable treatment modality. During the last thirty years, dental implants gained credit and popularity and showed improvements in all aspects.

Alberktsson et al. (1981) defined osseintegration as a direct structural and functional connection between ordered, living bone and the surface of the load carrying implant [19]. Key factors for successful and high level osseintegration included careful surgery, meticulous prosthodontics, and good after care, linked with good patient selection [20].

Table 3. Distribution of implants according to size and site.

\begin{tabular}{cccc}
\hline $\begin{array}{c}\text { Implant Size (Diameter } \\
\text { and Length) mm }\end{array}$ & $\begin{array}{c}\text { Number of Implants } \\
\text { in Maxillary Jaw }\end{array}$ & $\begin{array}{c}\text { Number of Implants } \\
\text { in Mandibular Jaw }\end{array}$ & Total \\
\hline $3.3 \times 10$ & 12 & 19 & 31 \\
$3.3 \times 11.5$ & 3 & 8 & 11 \\
$3.3 \times 13$ & 20 & 14 & 34 \\
$3.75 \times 10$ & 3 & 5 & 8 \\
$3.75 \times 11.5$ & 8 & 13 & 21 \\
$3.75 \times 13$ & 42 & 10 & 52 \\
$4.2 \times 10$ & 11 & 20 & 31 \\
$4.2 \times 11.5$ & 7 & 5 & 12 \\
$4.2 \times 13$ & 11 & 6 & 17 \\
Total & 117 & 100 & 217 \\
\hline
\end{tabular}

Table 4. The distribution of maxillary, mandibular and failed implants in each Jaw.

\begin{tabular}{ccc}
\hline Jaw & Number and percentage & Number and Percentage Of failed Implants \\
\hline Maxillary & $117(54 \%)$ & $6(3 \%)$ \\
Mandibular & $100(46 \%)$ & $4(2 \%)$ \\
Total & 217 & 10 \\
\hline
\end{tabular}


Table 5. Distribution of successful and failed implants according to size.

\begin{tabular}{cccc}
\hline $\begin{array}{c}\text { Implant Size } \\
\text { (Diameter and Length) mm }\end{array}$ & $\begin{array}{c}\text { Number of } \\
\text { Successful Implants }\end{array}$ & $\begin{array}{c}\text { Number of } \\
\text { Failed Implants }\end{array}$ & Total \\
\hline $3.3 \times 10$ & 23 & 8 & 31 \\
$3.3 \times 11.5$ & 11 & 0 & 11 \\
$3.3 \times 13$ & 34 & 0 & 34 \\
$3.75 \times 10$ & 7 & 1 & 8 \\
$3.75 \times 11.5$ & 21 & 0 & 21 \\
$3.75 \times 13$ & 52 & 0 & 52 \\
$4.2 \times 10$ & 31 & 0 & 31 \\
$4.2 \times 11.5$ & 11 & 1 & 12 \\
$4.2 \times 13$ & 17 & 0 & 17 \\
Total & 207 & 10 & 217 \\
\hline
\end{tabular}

Table 6. Oneway ANOVA to compare differences in implant failure according to Implant Parameters (length, diameter, and position).

\begin{tabular}{ccccc}
\hline Implant Parameter & Sum of Squares & Mean Square & F & Sig. \\
\hline Diameter & 1.026 & 1.026 & 4.368 & $0.038^{\star}$ \\
Length & 13.125 & 13.125 & 7.313 & $0.007^{\star}$ \\
Position & 0.028 & 0.028 & 0.123 & 0.727 \\
\hline
\end{tabular}

* significant when $P<0.05$.

Table 7. T test analysis to study the significance of patients parameters (age and gender) on failure of implants.

\begin{tabular}{cccccc}
\hline \multirow{2}{*}{ Patients' Parameters } & \multicolumn{2}{c}{ Coefficients } & \multirow{2}{*}{ Beta } & t & Sig. \\
\cline { 2 - 3 } & B & Std. Error & & & 0.114 \\
Age & 0.002 & 0.0021 & 0.100 & 1.587 & 0.23 \\
\hline Gender & 0.003 & 0.0029 & 0.112 & 1.688 & 0.12 \\
\hline
\end{tabular}

Attempts have been made to enhance the success rate of implants. Oral implant success rate can be enhanced by improving the surgical technique [21]. The future of osseintegrated implants depends on the comprehension of the important contributions by a responsible surgeon and restorative dentist [22]. From there, the concept of guided surgery appeared to enhance success and lessen patients' pain. Abu Hantash et al. (2006) found that pain consideration is a factor when assessing dental implant treatment acceptance and satisfaction [23]. Youk et al. (2014) showed that patients who have surgical implant therapy were more satisfied and gained acceptance than conventional implant technique due to safety and accuracy of surgical guide techniques [24].

The results of this study supported the effective and high success rates of guided implant surgery for medium and long implants (11.5 and 13) mm length and (3.75 and 4.2) mm diameter. These findings agreed with Lee et al. (2005) review who reported that wider and longer implants keep better success rates 
and prognosis in conventional implant surgical techniques [25].

There were no significant differences in success rates in relation of age, gender, and anatomic sites and guided implant surgery. On the contrary, previous studies [26-28] reported an early implant failure due to anatomical sites; especially anterior maxilla.

In addition, the results of this study showed that there was a significant difference between success rate of implants in guided surgery according to their sizes. Implant size $(3.3 \mathrm{~mm}$ diameter and $10 \mathrm{~mm}$ length) had more failure rates compared to other dimensions. This could be justified by the fact that the alveolar ridge in the implant site of small ones $(3.3$ and $10 \mathrm{~mm}$ ) was more atrophic and narrower than other sites if compared with other implant sites in the same patient and among different patients, also. High failure rate of small size implants as found in this study is best explained by the lack of control of the angle of drilling and implant placement blindly through the guide especially in the atrophic area of the alveolar ridges, which usually resulted in hidden and undiagnosed dehiscence or microfracture of alveolar bone which in future leads to more risk of infection, instability and failure.

Improving the prognosis of guided implant surgery of smaller sizes in atrophic bone could be achieved through two criteria: first, meticulous treatment planning and patient selection in regard to bone quality and quantity should be accomplished. Second, the need of open surgical instrumentation (drilling and insertion) in the area of atrophic ridges after making drill through the guide to ensure a proper implant insertion without chipping or future dehiscence. This could also be managed by inventing new guide implant systems designed carefully to avoid surgical complications in atrophic areas and ensure a gentler and more accurate placement of smaller implants.

\section{CONCLUSIONS}

The conclusion of this study can be summarized as the following:

1) Implants width $(3.3 \mathrm{~mm})$ and length $(10 \mathrm{~mm})$ are not recommended in guided computed tomography implant surgery as compared with other implant sizes.

2) Implant success rate is not correlated with patients' age, gender and anatomic sites.

3) Computer-guided implant surgery remains exciting. Watchful planning and precise stability of the surgical guide are mandatory for successful protocol.

4) Further investigations should be performed to assess such technique among dental patients from satisfactory and acceptance point of view.

\section{ACKNOWLEDGEMENTS}

The authors would like to thank Dr Haitham Al-Alami and Prof. Mahmoud Al-Omiri for their kind contribution in this study.

\section{CONFLICTS OF INTEREST}

The authors declare no conflicts of interest regarding the publication of this paper.

\section{REFERENCES}

1. Henry, P.J. and Liddelow, G.J. (2008) Immediate Loading of Dental Implants. Australian Dental Journal, 53, 569-581. https://doi.org/10.1111/j.1834-7819.2008.00044.x

2. Van Steenberghe, D., Glauser, R., Blombäck, U., et al. (2005) A Computed Tomographic Scan-Derived Customized Surgical Template and Fixed Prosthesis for Flapless Surgery and Immediate Loading of Implants in Edentulous Maxilla: A Prospective Multicenter Study. Clinical Implant Dentistry and Related Research, 7, S111-S120. https://doi.org/10.1111/j.1708-8208.2005.tb00083.x

3. Aparicio, C., Rangert, B. and Sennerby, L. (2003) Immediate/Early Loading of Dental Implants: A Report from the Sociedad Espańola de implantes worl Congress Consensus Meeting in Barcelona, Spain, 2002. Clinical Im- 
plant Dentistry and Related Research, 5, 57-60. https://doi.org/10.1111/j.1708-8208.2003.tb00183.x

4. Cochran, D.L., Morton, D. and Weber, H.P. (2004) Consensus Statements and Recommended Clinical Prodeures Regarding Loading Protocols for Endosseos Dental Implants. The International Journal of Oral \& Maxillofacial Implants, 19, 109-113.

5. Plalda, I., Smarandache, A.M. and Saban, M. (2012) Immediate Implant Loading. AMT, 2, 239-241.

6. Moraschini, V., Velloso, G., Luz, D. and Porto Barboza, E. (2015) Implant Survival Rates, Marginal Bone Less Changes and Complications in Full Mouth Rehabilitation with Flapless Computer-Guided Surgery: A Systemic Review and Meta-Analysis. International Journal of Oral and Maxillofacial Surgery, 44, 892-901.

7. Kalra, M., Aparna, W. and Dhanasekar, B. (2013) Evolution of Surgical Guidance in Implant Dentistry. Dental Update, 40, 577-578, 581-582. https://doi.org/10.12968/denu.2013.40.7.577

8. Mazor, Z., Segal, P. and Levin, L. (2015) Computer-Guided Implant Placement for Rehabilitation of the Edentulous Maxilla with Two Impacted Canines: An Approach without Extraction of the Impacted Teeth. The International Journal of Periodontics \& Restorative Dentistry, 35, 93-97. https://doi.org/10.11607/prd.2062

9. Youk, S.Y., Lee, J.H., Park, J.M., Heo, S.J., Roh, H.K., Park, E.J. and Shin, H. (2014) A Survey of the Satisfaction of Patients Who Undergone Implant Surgery with and without Employing a Computer Guided Implant Surgical Template. The Journal of Advanced Prosthodontics, 6, 395-405. https://doi.org/10.4047/jap.2014.6.5.395

10. Schiroli, G., Angiero, F., Zangerl, A., Bendicentu, S., Ferrante, F. and Widmann, G. (2015) Accuracy of Flapless Protocol for Computer-Guided Zygomatic Implant Placement in Human Cadavers: Expectations and Reality. International Journal of Medical Robotics, 12, 102-108. https://doi.org/10.1002/rcs.1646

11. Mandelaris, G.A. and Vik, S.D. (2014) Guided Implant Surgery with Placement of a Presurgical CAD/CAM Patient Specific Abutment and Provisional in the Esthetic Zone. Compendium of Continuing Education in Dentistry, 35, 494-504.

12. Vercruyssen, M., Hultin, M., Van Assche, N., Svensson, K., Naert, I. and Quiryen M. (2014) Guided Surgery: Accuracy and Efficacy. Periodontology 2000, 66, 228-246. https://doi.org/10.1111/prd.12046

13. Rathi, N., Scherer, M.D. and McGlumphy, E. (2014) Stabilization of a Computer-Aided Implant Surgical Guide Using Existing Dental Implants with Conversion of an Overdenture to a Fixed Prosthesis. Journal of Prosthodontics, 23, 634-638. https://doi.org/10.1111/jopr.12174

14. Doan, N.V., Du, Z., Reher, P. and Xiao, Y. (2014) Flapless Dental Implant Surgery: A Retrospective Study of a Consecutive Implant. The International Journal of Oral \& Maxillofacial Implants, 29, 650-658. https://doi.org/10.11607/jomi.3195

15. Mazor, Z., Segal, P. and Levin, L. (2015) Computer Guided Implant Placement for Rehabilitation of the Edentulous Maxilla with Two Impacted Canines: An Approach without Extraction of the Impacted Teeth. The International Journal of Periodontics \& Restorative Dentistry, 35, 93-97. https://doi.org/10.11607/prd.2062

16. Orentlicher, G., Goldsmith, D. and Abboud, M. (2012) Computer-Guided Planning and Placement of Dental Implants. Atlas of the Oral and Maxillofacial Surgery Clinics of North America, 20, 53-79.

https://doi.org/10.1016/j.cxom.2011.12.004

17. de Almeida, E.O., Pellizzer, E.P., Goiatto, M.C., et al. (2010) Computer-Guided Surgery in Implantology: Review of Basic Concepts. Journal of Craniofacial Surgery, 21, 1917-1921.

https://doi.org/10.1097/SCS.0b013e3181f4b1a0

18. Albrektsson, T., Zarb, G., Worthington, P. and Eriksson, R.A. (1986) The Long Term Efficacy of Currently Used Dental Implants: A Review and Propsed Criteria of Success. The International Journal of Oral \& Maxillofacial Implants, 1, 11-25.

19. Albrektsson, T., Brắnemark, P.I., Hansson, H.A. and Lindström, J. (1981) Osseointegrated Titanium Implants. 
Requirements for Ensuring a Long-Lasting, Direct Bone Anchorage in Man. Acta Orthopaedica Scandinavica, 52, 155-170. https://doi.org/10.3109/17453678108991776

20. Davis, M.D. (1998) The Shift in the Therapeutic Paradigm: Osseointegration. Journal of Prosthetic Dentistry, 79, 37-42. https://doi.org/10.1016/S0022-3913(98)70191-X

21. Albrektsson, T. (2001) Is Surgical Skill More Important for Clinical Success than Changes in Implant Hardware? Clinical Implant Dentistry and Related Research, 3, 174-175. https://doi.org/10.1111/j.1708-8208.2001.tb00138.x

22. Bryant, S.R. (2001) Oral Implant Outcomes Predicted by Age and Site for Specific Bone Condition. PhD Thesis, University of Toronto, Toronto.

23. Abu Hantash, R.O., AL-Omiri, M.K. and Al-Wahadni, A.M. (2006) Psychological Impact on Implant Patients' Oral Health Related Quality of Life. Clinical Oral Implants Research, 17, 116-123.

24. Youk, S.-Y., Lee, J.-H., Park, J.-M., Heo, S.-J., Roh, H.-K., Park, E.-J. and Shin, I.H. (2014) A Survey of the Satisfaction of Patients Who Have Undergone Implant Surgery with and without Employing a Computer-Guided Implant Surgical Template. The Journal of Advanced Prosthodontics, 6, 395-404. https://doi.org/10.4047/jap.2014.6.5.395

25. Lee, J.-H., Frias, V., Lee, K.-W. and Wright, R.F. (2005) Effect of Implant Size and Shape on Implant Success Rates: A Literature Review. Journal of Prosthetic Dentistry, 94, 377-381. https://doi.org/10.1016/j.prosdent.2005.04.018

26. Fouda, A.A. (2020) The Impact of the Alveolar Bone Sites on Early Implant Failure: A Systematic Review with Meta-Analysis. Journal of the Korean Association of Oral and Maxillofacial Surgeons, 46, 162-173. https://doi.org/10.5125/jkaoms.2020.46.3.162

27. Lang, L.A., Hansen, S.E., Olvera, N. and Teich, S. (2019) A Comparison of Implant Complications and Failures between the Maxilla and the Mandible. Journal of Prosthetic Dentistry, 121, 611-617. https://doi.org/10.1016/j.prosdent.2018.08.002

28. Baqain, Z.H., Moqbel, W.Y. and Sawair, F.A. (2012) Early Dental Implant Failure: Risk Factors. British Journal of Oral and Maxillofacial Surgery, 50, 239-243. https://doi.org/10.1016/j.bjoms.2011.04.074 